\title{
Modification of the secondary structure of angiotensin II by substitution of hydrogen with Cs cations: an experimental and theoretical study
}

\author{
J. M. Wichmann, ${ }^{a}$ R. Mitrić, ${ }^{b}$ C. Weise, ${ }^{c}$ M. Holz ${ }^{a}$ and A. Lindinger ${ }^{* a}$ \\ Received 5th September 2011, Accepted 5th December 2011 \\ DOI: $10.1039 / \mathrm{c} 2 \mathrm{cp} 22808 \mathrm{~d}$
}

MALDI mass spectrometry in combination with post-source decay (PSD) analysis is a fast and easy to apply method for peptide sequencing. In this study, the PSD technique was used to investigate the influence of the adaption of one, two, and three caesium cations to angiotensin II in the gas phase. The PSD spectra of caesium-aggregated angiotensin II show far less fragmentation in comparison to the protonated one. In the case of singly (doubly) $\mathrm{Cs}^{+}$substituted angiotensin II, the PSD mass spectrum shows only fragments with one (two) Cs cation(s). These results are interpreted in terms of additional interactions of the caesium cation(s) with the peptide. In order to investigate this suggestion, the molecular structures were calculated with semi-empirical molecular dynamic (MD) simulations and further optimized at the quantum chemical level (BP86, SVP) of theory. On the one hand, secondary structures of $\mathrm{Cs}^{+}$substituted angiotensin II are more compact than the structure of protonated angiotensin II, indicating electrostatic interactions of the Cs cations and the heterocyclic structures. Moreover, oxyphilic interactions of the cations with the oxygen atoms of the peptide backbone also contribute as further van-der-Waals interactions of the $\mathrm{Cs}^{+}$substituted angiotensin II. These interactions are able to explain its higher stability due to reduced dissociation in comparison to the protonated angiotensin II. On the other hand, most MD simulations of doubly and triply $\mathrm{Cs}^{+}$substituted angiotensin II show a formation of a [2 Cs] cluster, surrounded by the peptide molecule. The formation of this cluster would explain the lack of singly $\mathrm{Cs}^{+}$substituted fragments in the PSD mass spectrum of doubly $\mathrm{Cs}^{+}$substituted angiotensin II.

\section{Introduction}

Since the invention of matrix-assisted laser desorption/ionization (MALDI) in the 1980s by M. Karas, F. Hillenkamp in Germany and by K. Tanaka and co-workers in Japan, large fragile molecules can be transferred into the gas phase. ${ }^{1-5}$ In combination with mass spectrometry (MS), MALDI-MS became one of the most important mass analysis techniques in biochemistry, physiology and polymer science. Due to the high mass of typical bio-macromolecular analyte molecules, a reflectron type of a time of flight (TOF) analyzer is built into most commercial MALDI-TOF devices. After desorption a small fraction of the (mostly singly) charged analyte molecules decays on their way to the detector. Mass spectrometric analysis of these generated fragments from metastable decay can provide information about the molecular structure of the analyte molecule (in the case of peptides: the amino-acid sequence). ${ }^{6-8}$ This technique is known under the name of post-source decay (PSD) analysis.

\footnotetext{
${ }^{a}$ Freie Universität Berlin, Institut für Experimentalphysik, Arnimallee 14, 14195, Berlin, Germany.E-mail: albrecht.lindinger@fu-berlin.de

${ }^{b}$ Freie Universität Berlin, Institut für Theoretische Physik, Arnimallee 14, 14195, Berlin, Germany

${ }^{c}$ Freie Universität Berlin, Institut für Chemie und Biochemie,

Thielallee 63, 14195, Berlin, Germany
}

By using the fragment scheme introduced by Roepstorff and Fohlmann in combination with the detected $\mathrm{m} / \mathrm{z}$ values of the fragments, the primary structure of a small peptide (up to a molecular mass of about $3000 \mathrm{Da}$ ) can be easily obtained. ${ }^{9}$ In modern MALDI-MS devices, developments like the tandem mass spectrometer (MALDI-TOF-TOF) are able to produce PSD-MALDI mass spectra of high quality within seconds of experimental effort. ${ }^{10}$ By using this technique, the decay of a defined analyte (A) ion (e.g. $\left.[\mathrm{A}+\mathrm{H}]^{+}\right)$could be examined exclusively. The cleavage of the $-\mathrm{CO}-\mathrm{NH}-$ bond in the peptide backbone yields $\mathrm{y}$ - and b-ions that can be detected with high signal intensity during PSD-MALDI mass analysis. In general, MALDI mass spectra contain $[\mathrm{A}+\mathrm{Na}]^{+}$or $[\mathrm{A}+\mathrm{K}]^{+}$mass peaks as well as the protonated one $[\mathrm{A}+\mathrm{H}]^{+}$due to contamination of the MALDI sample. ${ }^{11-13}$ If the analyte is substituted with a doubly charged cation or more than one singly charged cation, the analyte becomes deprotonated, until it is singly positively charged.

A key factor for the activity of biologically relevant molecules is their secondary structure. Additional cations could change the secondary structure of peptides, as we will show in this paper. The ratio of cation substitution of the analyte A (i.e. $[\mathrm{A}+\mathrm{Na}]^{+} /[\mathrm{A}+\mathrm{H}]^{+}$) in MALDI mass spectra strongly increases with the absorbed laser pulse energy, as shown in 
former studies for angiotensin II with potassium. ${ }^{14}$ Additionally MALDI experiments with high laser pulse energies suggest a higher stability of angiotensin II, if alkali metal ion(s) is (are) attached. ${ }^{15}$ Nevertheless, the bonding position and resulting changes of the molecular structure of peptides by substitution with metal cations are not completely analyzed in the gas phase.

For the present examination, the analyte is angiotensin II $\left(\mathrm{H}_{2} \mathrm{~N}\right.$-Asp-Arg-Val-Tyr-Ile-His-Pro-Phe-COOH; $\left.m=1046.2 \mathrm{Da}\right)$. On the one hand, angiotensin II (Ang) is regarded as a model system due to the easy detection using MALDI mass spectrometry. On the other hand, the peptide plays a vital role in several cardiological processes like regulation of the blood pressure and salt homeostasis in human metabolism. ${ }^{16,17}$ The investigation of the $\mathrm{Cs}^{+}$-substituted form of the hormone angiotensin II could be relevant in a medical context, as some studies suggest. ${ }^{18}$ Therefore, gas-phase examinations could be a step to understand the influence of cation interaction with various biomolecules, also in solution phase.

According to experiments by Sullards and Reiter, the additional proton can be located at the side chains of the amino acids - arginine, histidine, or the carbonyl oxygen atoms of the peptide backbone. ${ }^{19}$ Due to the basicity of the arginine residue, a protonation of this residue seems highly favourable. These positions could also be preferred by small cations such as lithium or sodium. The bonding of alkali metal cations to peptides and other biologically relevant molecules has been studied extensively, but it is still discussed as recent reports suggest. ${ }^{20-22}$ However, many of these are either theoretical or experimental. Our combined study deals with the direct influence of caesium cations on the structure of angiotensin II.

In this work, the change of the secondary structure of protonated and $\mathrm{Cs}^{+}$-substituted angiotensin II has been examined by using PSD-MALDI mass spectrometry for the detection of the fragments of $[\mathrm{Ang}+\mathrm{H}]^{+},[\mathrm{Ang}+\mathrm{Cs}]^{+},[\mathrm{Ang}+2 \mathrm{Cs}-\mathrm{H}]^{+}$, and $[\mathrm{Ang}+3 \mathrm{Cs}-2 \mathrm{H}]^{+}$. The results are interpreted with regard to their molecular structures. For that purpose, molecular dynamics (MD) simulations on the semi-empirical level of theory have been performed. In comparison to commonly used classical MD simulations (using force fields), quantum chemical approaches are able to describe the changes in the electronic structure such as bond cleavage or formation. For higher accuracy of the results of the MD simulations, their geometries have been further optimized by density functional theory (DFT).

\section{Experimental}

\subsection{Experimental setup}

All PSD-MALDI mass spectra have been generated with a commercial MALDI mass spectrometer (Ultraflex II, Bruker Daltonics, Bremen, Germany). The MALDI samples have been irradiated with a frequency-tripled Nd-YAG laser $(\lambda=355 \mathrm{~nm}$, $\tau=5 \mathrm{~ns}$ ). For obtaining high-quality PSD mass spectra, the LIFT method has been employed. ${ }^{10}$ The analyte ions, produced during MALDI are accelerated into a linear TOF. After the first TOF, ions of a defined $m / z$-value were analyzed in a second reflectron-type TOF. This tandem mass spectrometry technique allows the measurement of a complete PSD mass spectrum in one step. For further data processing, the program package
FlexAnalysis 2.4 (Bruker Daltonics) has been applied. A mixture containing $\alpha$-cyano-4-hydroxycinnamic acid (CHCA) and sinapic acid (SA) (about $10 \mathrm{mg} \mathrm{ml}^{-1}$ each in $3: 2$ acetonitrile/water) was used as a matrix for the MALDI experiments. Caesium chloride and angiotensin II were prepared in dest. $\mathrm{H}_{2} \mathrm{O}$ (about $50 \mu \mathrm{g} \mathrm{ml}^{-1}$ each). This combination generated a high degree of cationization and also strong fragmentation of the peptide under MALDI conditions. Samples were spotted on the MALDI target plates using the dried droplet method.

\subsection{Theoretical approach}

The exploration of potential energy surfaces of protonated and cation-substituted angiotensin II has been performed using the simulated annealing procedure combined with molecular dynamics simulations at the semi-empirical AM1 level of theory. ${ }^{23}$ For electronic structure calculations, the MOPAC program package has been used and MD simulations have been performed with an in-house suite of programs. ${ }^{24}$ The simulations were started typically at $T=1000 \mathrm{~K}$ and the system has been exponentially cooled down to $T=0 \mathrm{~K}$ within $10 \mathrm{ps}$ of simulation time. For all structures, several simulated annealing runs have been carried out by varying the simulation parameters such as initial structure and cooling time. The structures obtained from the simulated annealing procedure have been further optimized using DFT (with RI approximation). For this purpose the gradient corrected BP86 functional together with single split valence plus polarization (SVP) basis sets have been employed together with the relativistic effective core potential (ECP) for the Cs atom. ${ }^{25-30}$ All DFT calculations have been performed using the ORCA (version 2.7) package. $^{31}$

\section{Results and discussion}

This section is separated into four parts. The first part describes the PSD mass spectrum and the molecular structure of protonated angiotensin II $\left([\mathrm{Ang}+\mathrm{H}]^{+}\right)$. The three other parts report on the singly $\left([\mathrm{Ang}+\mathrm{Cs}]^{+}\right)$, doubly $\left([\mathrm{Ang}+2 \mathrm{Cs}-\mathrm{H}]^{+}\right)$, and triply $\left([\mathrm{Ang}+3 \mathrm{Cs}-2 \mathrm{H}]^{+}\right) \mathrm{Cs}^{+}$-substituted angiotensin II.

For convenience of expression we chose to denote the replacement of a single proton by a $\mathrm{Cs}^{+}$ion "caesiation" (in analogy with deuteration) and the substituted peptide "caesiated".

\subsection{Protonated angiotensin II}

PSD mass spectra of protonated angiotensin II are well known. ${ }^{32,33}$ Main fragments are $y$ - and b-ions. Our PSD mass spectrum shows slightly higher signals for fragments near the termini (see Fig. 1).

The result of a DFT optimized calculation is shown in Fig. 1 for the uncharged (left) and protonated (right) molecular structure. As a reference configuration for all calculations of angiotensin II, a structure from the Protein Data Base (1N9V) has been used. The backbone of the uncharged molecule appears in a rather linear form (sort of a stretched helix), besides the bend resulting from valine.

The calculated molecular structure of $[\mathrm{Ang}+\mathrm{H}]^{+}$shows a mainly linear structure of the backbone as well as the uncharged molecule. Only minor modifications due to electrostatic interaction of the additional proton can be observed. The results of various simulations suggest a rather low influence of the 

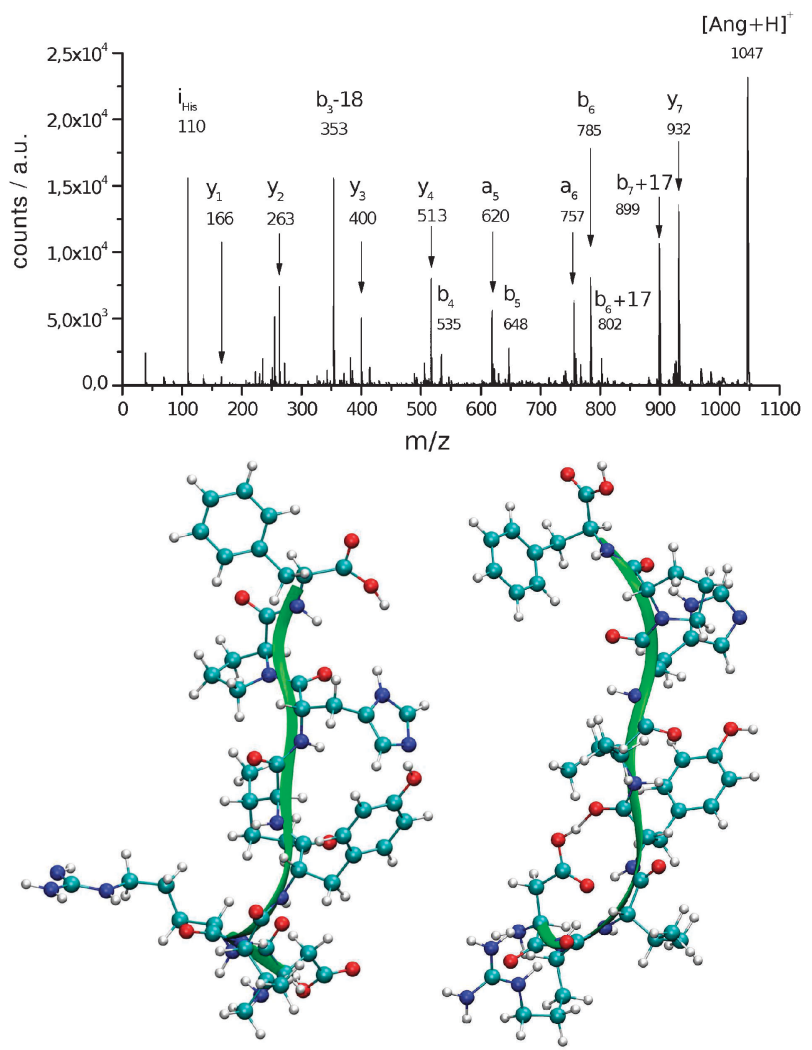

Fig. 1 In the upper part of the figure, the PSD MALDI mass spectrum of protonated angiotensin II is shown. The peak intensities of the fragments are in the same range as the $[\mathrm{Ang}+\mathrm{H}]^{+}$mass peak. The numbers refer to the $m / z$ value of the fragments. The ion intensity of fragments in the middle mass range is slightly lower. In the lower part of the figure, the unprotonated (left) and the protonated (right) angiotensin II structures are shown. The additional proton in the right structure is located at the arginine residue. The green ribbon indicates the backbone of the peptide.

additional proton on the secondary structure and the stability of the molecule in the gas phase. Therefore, we present the structure where the additional proton is located at the arginine residue. Experimentally, this result is in agreement with the rather high gas-phase fragmentation abundance, as shown in the corresponding PSD mass spectrum.

\subsection{Singly caesiated angiotensin II}

The PSD mass spectrum of singly caesiated angiotensin II $\left([\mathrm{Ang}+\mathrm{Cs}]^{+}\right)($Fig. 2) shows distinctly less fragmentation than the protonated peptide. For better visibility, the range of $m / z$ of 200 to 1000 has been enlarged. Shifted fragments (mostly $\mathrm{y}$-fragments and some $\mathrm{a}$ - and b-fragments) were obtained with a low ion signal. Mass peaks with a $m / z$ value lower than 400 (e.g. $\left[\mathrm{y}_{1}+\mathrm{Cs}\right]^{+}$or $\left.\left[\mathrm{y}_{2}+\mathrm{Cs}\right]^{+}\right)$, are below the noise level. The lower level of fragmentation indicates a higher stability of the oligopeptide protecting it from metastable decay in the reflectronTOF measurement. Uncaesiated fragments could not be identified.

The calculated secondary structure of $\mathrm{Cs}^{+}$-substituted angiotensin II shows a strong change of the secondary structure because of interaction between the (hetero-) cyclic side chains and the (centered) Cs cation. Some simulations also show an

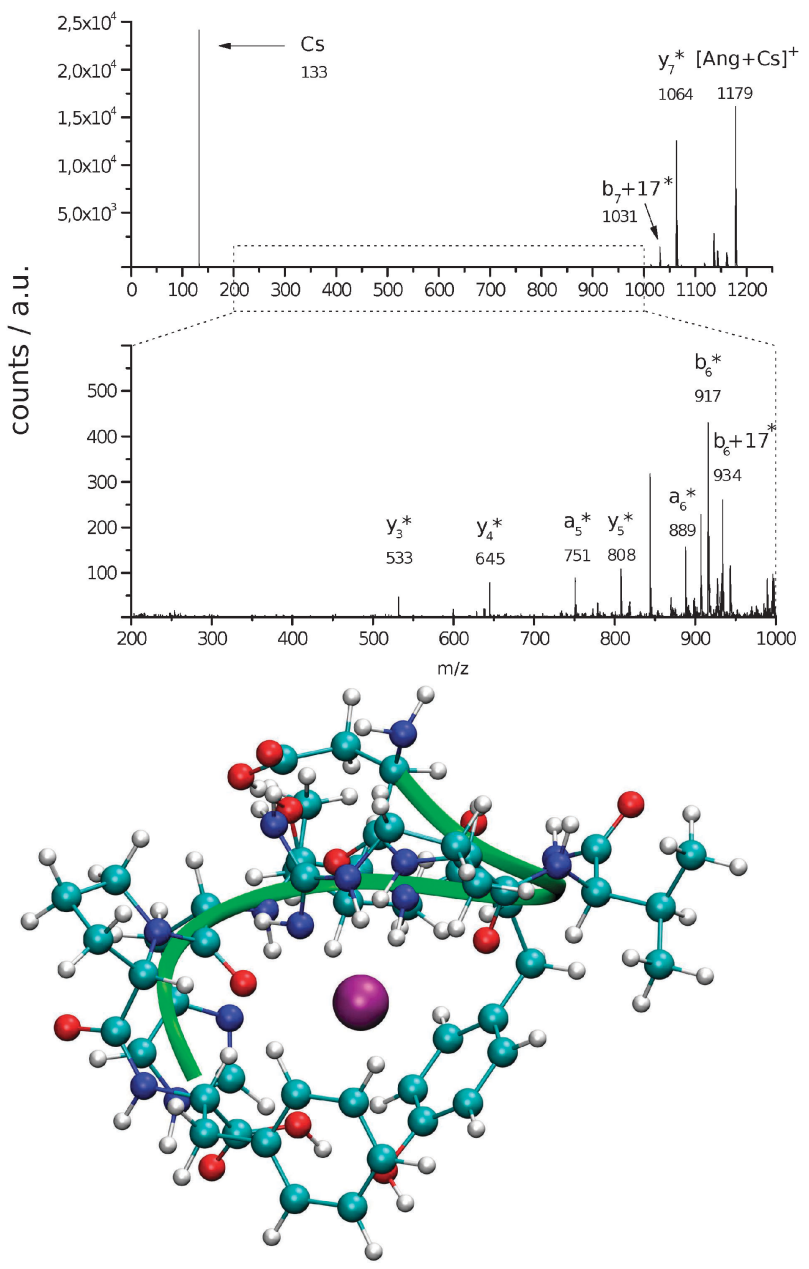

Fig. 2 The PSD mass spectrum of singly caesiated angiotensin II $[\mathrm{Ang}+\mathrm{Cs}]^{+}$shows only minor fragmentation. Therefore, the inner region ( $m / z$ from 200 to 1000) of the mass spectrum has been enlarged in the PSD mass spectra (* denotes the singly caesiated $\mathrm{y}_{i}$ fragment). The lower part shows a possible molecular structure of $[\mathrm{Ang}+\mathrm{Cs}]^{+}$. The Cs cation (purple) is surrounded by the peptide in the form of a cage.

interaction of the oxygen atoms of the peptide backbone with the cation and could be described as van-der-Waals interactions of the polarized oxygen atoms and the Cs cation. However, these interactions seem to be weaker than the interaction of the cations and the heterocyclic residues (e.g. tyrosine or histidine). The interactions change the structure such that the peptide forms a cage-like structure around the cation, as shown in Fig. 2. The main reason for this seems to be the interaction of the cation and the arginine/aspargine, the aromatic ring of the tyrosine and phenylalanine and the heterocyclic rest of the histidine. The delocalized $\pi$-systems of (ring) structures build up an attractive interaction with charged particles, here $\mathrm{Cs}^{+}$cations. ${ }^{34}$ This attractive interaction seems to be the main stabilizing factor of the molecular structure preventing it from fragmentation during mass spectrometry. The lack of several fragments, and the increased intensity of some $y$ - and b-fragment ion signals with higher fragment size indicate a stable position of the substituted cation and its interaction with the peptide. In particular, the high ion signal of the $\mathrm{y}_{7}$-fragment could be the result of the higher basicity of the fragment in comparison to 
the peptide due to the loss of the acidic Asp residue. The substantial ion signal of the $b_{7}-17$ fragment is in agreement with reaction schemes presented by Grese et al. and Teesch et al. (and references therein). ${ }^{20,21}$ These studies suggest that the cation attacks the $\mathrm{C}$-terminus and the peptide loses $\mathrm{a}-\mathrm{C}=\mathrm{O}$ and an amino group with the residue nearby. A protonation/ deprotonation of single amino-acid residues shows a rather low influence on the secondary structure if caesium is attached. The absence of protonated fragments also suggests a strong bonding of the cation to the peptide and an inhibition of charge transport inside the analyte molecule. A possible reason could be the size/mass of the Cs cation.

\subsection{Doubly caesiated angiotensin II}

This molecule shows more fragmentation than $[\mathrm{Ang}+\mathrm{Cs}]^{+}$, as displayed in the PSD mass spectrum of $[\mathrm{Ang}+2 \mathrm{Cs}-\mathrm{H}]^{+}$(see Fig. 3). Here the $m / z$ values of the $y$-fragments are shifted by $[2 \mathrm{Cs}-\mathrm{H}]=265 \mathrm{Da}$. Also in this case, mostly $\left[\mathrm{y}_{i}+2 \mathrm{Cs}\right]^{+}$fragments have been detected. Additionally, there are some mass peaks which could not be assigned. The molecular structure of $[\mathrm{Ang}+2 \mathrm{Cs}-\mathrm{H}]^{+}$is shown in Fig. 3. The structure
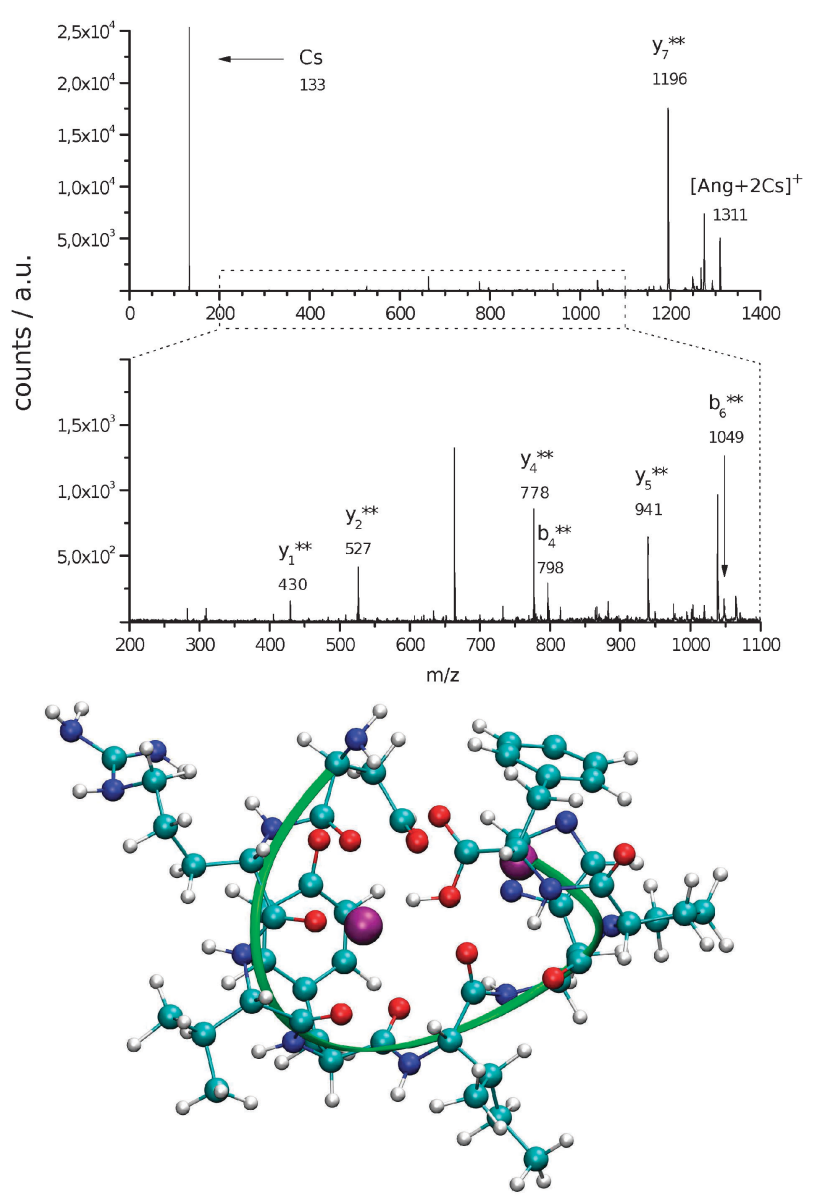

Fig. 3 In the upper part of this figure, the PSD mass spectrum of doubly caesiated angiotensin II is shown. There is only minor fragmentation of $[\text { Ang }+2 \mathrm{Cs}-\mathrm{H}]^{+}$in the gas phase. Most fragments are y fragments. $\mathrm{y}_{i}{ }^{* *}$ stands for $\left[\mathrm{y}_{i}+2 \mathrm{Cs}-\mathrm{H}\right]$. Every identified fragment is doubly caesiated. The lower part of this figure shows a geometrically optimized structure of $[\text { Ang }+2 \mathrm{Cs}-\mathrm{H}]^{+}$. Both Cs atoms are nearby and surrounded by (hetero) cyclic residues. shows both Cs atoms/cations in a cage of the peptide. Some MD simulations show a separation of the Cs atom(s)/cation(s) by a cyclic amino acid residue. In almost all calculated structures, the Cs atom(s)/cation(s) are surrounded by the ring structures of the tyrosine, phenylalanine and histidine side chains. MD simulations suggest that the protonation/ deprotonation of single amino acids is not crucial for the secondary structure of $\mathrm{Cs}^{+}$-substituted angiotensin II. This result can also be interpreted by the increased interaction of $\mathrm{Cs}$ cations with the peptide compared to the interaction of the peptides with an additional proton. The lack of singly caesiated fragments in the mass spectra indicates the formation of the [2Cs] cation inside the peptide cage. Also in the case of the doubly caesiated angiotensin II, the molecular structure seems to be stabilized by electrostatic interaction of the cations and the (deprotonated) peptide.

\subsection{Triply caesiated angiotensin II}

In MALDI mass spectra, the peak intensity of $[\mathrm{Ang}+3 \mathrm{Cs}-2 \mathrm{H}]^{+}$ is rather low in comparison to the other analyte peak intensities. One reason could be the high reaction barrier of the proton/ cation exchange reaction during the MALDI process. Also the PSD mass spectra of $[\mathrm{Ang}+3 \mathrm{Cs}-2 \mathrm{H}]^{+}$(shown in Fig. 4) exhibit low signal intensities in comparison to the protonated or the singly caesiated species. This low ion signal is in agreement with former results. ${ }^{14}$ Since the ratio of the fragment peak intensities and the $[\mathrm{Ang}+3 \mathrm{Cs}-2 \mathrm{H}]^{+}$is rather high, this structure seems to be less stable than the singly and doubly $\mathrm{Cs}^{+}$-substituted angiotensin II. In the PSD mass spectrum there are several fragments, which cannot be assigned to caesiated fragments. The fragment peak intensities are in the same range as the analyte
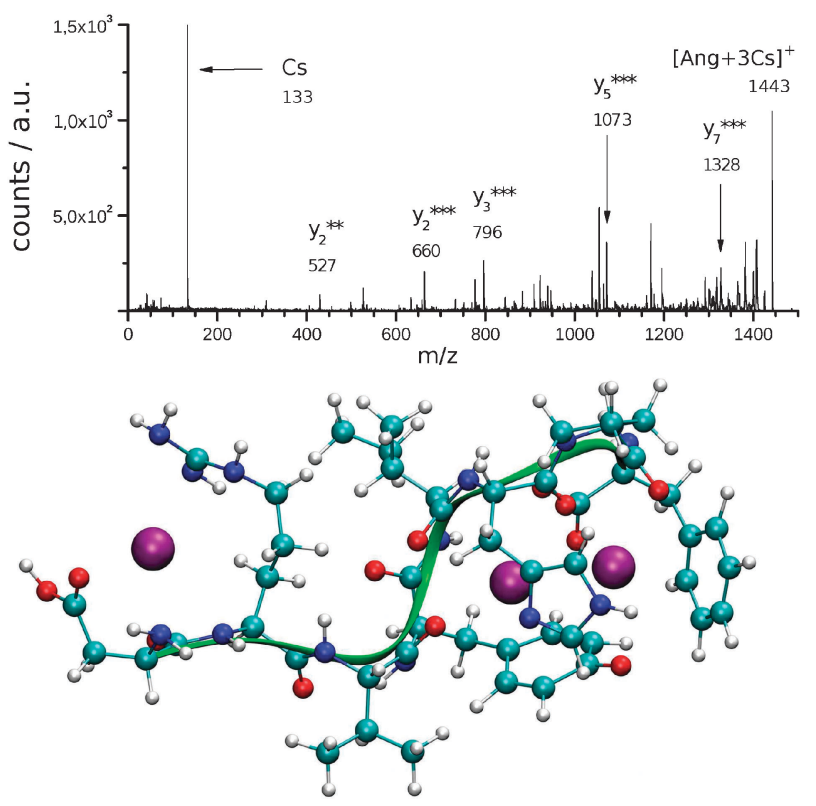

Fig. 4 The PSD mass spectrum of [Ang + 3Cs-2H] shows rather high fragmentation in comparison to the other caesiated species, yet many peaks could not be identified. Besides triply caesiated fragments (denoted with ***) there is also one doubly caesiated fragment (denoted by **). The lower part shows the geometrically optimized structure of [Ang $+3 \mathrm{Cs}-2 \mathrm{H}]$. Two of the Cs ions form a cluster and one Cs atom/ion is located nearby. 
peak intensity. In contrast to the other PSD mass spectra, there is a fragment which contains only two Cs atoms instead of three.

The calculated molecular structure is likely a [2Cs] dimer structure and one separated $\mathrm{Cs}$ atom/cation as several MD simulations suggest. This $\mathrm{Cs}$ dimer could explain the $\left[\mathrm{y}_{2}+2 \mathrm{Cs}\right]^{+}$ fragment. The molecular structure also suggests an interaction between the $\mathrm{Cs}^{+}$cation(s)/atom(s) and the cyclic structures of tyrosine, phenylalanine, histidine, and the deprotonated terminus. This seems to be the main factor for stabilization of the triply caesiated angiotensin II. The molecular structure in this case suggests a similar interaction between the cluster and the heterocyclic (or polar) amino acid residues. Repulsive interaction between the caesium cations could contribute to increased peptide fragmentation of the mainly linear peptide backbone. This repulsion can be seen as a reason for the higher fragmentation rate of $[\mathrm{Ang}+3 \mathrm{Cs}-2 \mathrm{H}]^{+}$. Also the $\left[\mathrm{y}_{2}+2 \mathrm{Cs}\right]^{+}$fragment indicates the repulsive interaction of the $\mathrm{Cs}$ atom/cation and the Cs dimer located close to each other.

\section{Conclusion}

The PSD mass spectra of caesiated angiotensin II have been presented in combination with the calculated molecular structures. PSD analysis of caesiated angiotensin II shows a very low fragmentation rate of caesiated angiotensin II in comparison to the protonated species. This suggests a higher gas phase stability of caesiated angiotensin II than the protonated one. In order to explain the experimental results, the molecular structures have been calculated. An important feature is the strong modification of the secondary structure of cationized angiotensin II in comparison to its protonated form. A more compact structure with additional interactions could lead to minor fragmentation of caesiated angiotensin II. Fragments of $[\mathrm{Ang}+\mathrm{Cs}]^{+}$and $[\mathrm{Ang}+2 \mathrm{Cs}-\mathrm{H}]^{+}$only show (Cs or $2 \mathrm{Cs}-\mathrm{H}$, respectively) shifted fragments. Most calculated structures suggest that cations interact with the (delocalized) $\pi$-systems of the cyclic amino acid residues-tyrosine, histidine or phenylalanine. This interaction seems to be stronger than the van-der-Waals interaction of the cation and the polar oxygen atoms of the peptide backbone (oxyphilic interaction) since only a few calculated structures stay in such a position. These further interactions change the secondary structure of the peptide considerably and increase the gas-phase stability of the caesiated peptides in comparison to the protonated angiotensin II. This is also in agreement with results of Dougherty and co-workers, who examined in detail the interaction of charged particles and (hetero-) cyclic structures. ${ }^{34}$ For higher caesiated species, especially in the case of $[\text { Ang }+3 \mathrm{Cs}-2 \mathrm{H}]^{+}$, the caesium cations/dimer could also interact repulsively. This could explain the lower stability of the triply cationized peptide in the gas phase as the higher fragmentation rate suggests, visible in the PSD mass spectrum of [Ang $+3 \mathrm{Cs}-2 \mathrm{H}]$. The substitution with other cations such as potassium or rubidium in angiotensin II shows similar effects (not shown here). These results transferred to systems in solution could give a hint as to how cation concentrations in blood could influence the effectiveness of hormones in a metabolic context. The finding that the interaction of the cyclic structures and the cations is more important than the precise positions of the protons at the peptide could show a way to interpret the relevant factor of salt concentration in biological (metabolic) systems.

\section{Acknowledgements}

The authors would like to thank Ludger Wöste for enthusiastically initiating the research direction, constant friendly encouragement and fruitful discussions. The IT service of the physics department of the Freie Universität is acknowledged for CPU time and HD space on their cluster and further help and the SFB 450 and the Dahlem Research School for financial support.

\section{References}

1 M. Karas, D. Bachmann and F. Hillenkamp, Anal. Chem., 1985, 57, 2935-2939.

2 M. Karas and U. Bahr, Trends Anal. Chem., 1986, 5, 90-93.

3 M. Karas, D. Bachmann, U. Bahr and F. Hillenkamp, Int. J. Mass Spectrom., 1987, 78, 53-68.

4 M. Karas and F. Hillenkamp, Anal. Chem., 1988, 60, 2299-2301.

5 K. Tanaka, H. Waki, Y. Ido, S. Akita, Y. Yoshida, T. Yoshida and T. Matsuo, Rapid Commun. Mass Spectrom., 1988, 2, 151-153.

6 B. Spengler, D. Kirsch and R. Kaufmann, J. Phys. Chem., 1992, 96, 9678-9684.

7 B. Spengler, D. Kirsch and R. Kaufmann, Rapid Commun. Mass Spectrom., 1991, 5, 198-202.

8 B. Spengler, J. Mass Spectrom., 1997, 32, 1019-1036.

9 P. Roepstorff and J. Fohlman, Biol. Mass Spectrom., 1984, 11, 601.

10 D. Suckau, A. Resemann, M. Schuerenberg, P. Hufnagel, J. Franzen and A. Holle, Anal. Bioanal. Chem., 2003, 376, 952-965.

11 J. F. Leite, M. R. Hajivandi, T. Diller and R. M. Pope, Rapid Commun. Mass Spectrom., 2004, 18, 2953-2959.

12 C. K. L. Wong and T. W. D. Chan, Rapid Commun. Mass Spectrom., 1997, 11, 513-519.

13 R. Knochenmuss, E. Lehmann and R. Zenobi, Eur. J. Mass Spectrom., 1998, 4, 421-427.

14 J. Wichmann, C. Lupulescu, L. Wöste and A. Lindinger, Eur. Phys. J. D, 2009, 52, 151-154.

15 J. M. Wichmann, F. Schwaneberg, C. Lupulescu and A. Lindinger, Int. J. Mass Spectrom., 2010, 296, 30-35.

16 M. Paul, A. P. Mehr and R. Kreutz, Physiol. Rev., 2006, 86, 747-803.

17 D. J. Voet and J. G. Voet, Biochemistry, John Wiley \& Sons, 4th edn, 2010, p. 547.

18 N. Gondo, K. Kumagai, H. Nakashima and K. Saku, Cardiovasc. Res., 2001, 49, 381-390.

19 M. C. Sullards and J. A. Reiter, J. Am. Soc. Mass Spectrom., 2000, 11, 40-53.

20 R. P. Grese, R. L. Cerny and M. L. Gross, J. Am. Chem. Soc., 1989, 111, 2835-2842.

21 L. M. Teesch, R. C. Orlando and J. Adams, J. Am. Chem. Soc., 1991, 113, 3668-3675.

22 D. Semrouni, C. Clavaguera, J.-P. Dognon and G. Ohanessian, Int. J. Mass Spectrom., 2010, 297, 152-161.

23 M. J. S. Dewar and W. Thiel, J. Am. Chem. Soc., 1977, 15, 4899-4907.

24 J. Stewart, MOPAC, 2001.

25 A. D. Becke, Phys. Rev. A, 1988, 38, 3098-3100.

26 J. P. Perdew, Phys. Rev. B, 1986, 33, 8822-8824.

27 P. J. Hay and W. R. Wadt, J. Chem. Phys., 1985, 82, 270-283.

28 D. Feller, J. Comput. Chem., 1996, 17, 1571-1586.

29 K. L. Schuchardt, B. T. Didier, T. Elsethagen, L. Sun, V. Gurumoorthi, J. Chase, J. Li and T. L. Windus, J. Chem. Inf. Model., 2007, 47, 1045-1052.

30 A. Hellweg, S. H. C. Hättig and W. Klopper, Theor. Chem. Acc., 2007, 117, 587-597.

31 F. Neese, ORCA-an ab initio, DFT and Semiempirical electronic structure package, 2010, Version 2.7.

32 J. Jai-nhuknan and C. J. Cassady, Rapid Commun. Mass Spectrom., 1996, 10, 1678-1682.

33 M. M. Cordero, T. J. Cornish, R. J. Cotter and I. A. Lys, Rapid Commun. Mass Spectrom., 1995, 9, 1356-1361.

34 J. C. Ma and D. A. Dougherty, Chem. Rev., 1997, 97, 1303-1324. 